\title{
Very high energy gamma-emission of Perseus Cluster
}

\author{
V.G. Sinitsyna* \\ P.N. Lebedev Physical Institute, Leninsky pr. 53, Moscow, Russia \\ E-mail: sinitsasci.lebedev.ru

\section{V.Y. Sinitsyna} \\ P.N. Lebedev Physical Institute, Leninsky pr. 53, Moscow, Russia \\ E-mail: sinits@sci.lebedev.ru
}

\begin{abstract}
The cluster of galaxies in Perseus have long been considered as possible candidates for the sources of high and very high energy $\gamma$-ray emission generated by various mechanisms. The Seyfert galaxy NGC 1275 is the central, dominant galaxy in the Perseus cluster of galaxies. NGC 1275 is known as a powerful source of radio and X-ray emission. The well-known extragalactic object NGC 1275 has been observed by the SHALON high-altitude mirror Cherenkov telescopes as a part of program of long-term studies of metagalactic gamma-ray sources. In 1996, the SHALON observations revealed a new metagalactic source of very high energy gamma-ray emission coincident in its coordinates with the galaxy NGC 1275. Having analyzed the SHALON data, we have determined such characteristics of NGC 1275 as the spectral energy distributions and images at energies $>800 \mathrm{GeV}$ for the first time. The results obtained at very high energies by SHALON are indicate that a part of $\mathrm{TeV} \gamma$-ray emission is generated by relativistic jets in the nucleus of NGC 1275 itself, but the presence of an extended structure around NGC 1275 is evidence of the interaction of cosmic rays and magnetic fields generated in the jets at the galactic center with the gas of the Perseus cluster. Also, we present the results of long-term observations of the Perseus Cluster which are revealed the $\gamma$-ray emission from the two of nearby object - GK Per of classical nova type, located at $\sim 3^{\circ} \mathrm{SW}$ from NGC 1275 and the explosion of extragalactic supernova SN2006gy that is about 10 minutes away from NGC 1275. So, the explosion of extragalactic supernova was observed at TeV energies for the first time with SHALON Cherenkov telescope.
\end{abstract}

Frontiers of Fundamental Physics 14 - FFP14,

15-18 July 2014

Aix Marseille University (AMU) Saint-Charles Campus, Marseille

\footnotetext{
*Speaker.
} 


\section{Introduction}

The cluster of galaxies in Perseus is one of the best-studied clusters owing to its relative proximity (its distance $\sim 100 \mathrm{Mpc}$ or redshift $\mathrm{z}=0.0179$ ) and brightness. Clusters of galaxies have long been considered as possible candidates for the sources of $\mathrm{TeV}$ gamma rays emitted by protons and electrons accelerated at large-scale shocks or by a galactic wind or active galactic nuclei $[1,2,3,4,5,6,7,8,9]$. The dominant galaxy in the Perseus cluster is NGC 1275 (Figs.1,2,3).

NGC 1275 is a powerful source of radio and X-ray emission. The NGC 1275 surrounded by extended filamentary structures historically aroused great interest owing to both its position at the center of the Perseus cluster and its possible "feedback" role [10]. Evidence for the "feedback" role of NGC 1275 can be obtained from ROSAT and Chandra observations, which reveal shells of hot gas and cavities that spatially coincide with the radio structures (Fig. 3) extending from the central, active part of the AGN. NGC 1275 also arouses interest owing to its close proximity to the Earth at redshift $\mathrm{z}=0.0179$ [11], making it possible to study the physics of relativistic jets.

\section{NGC 1275 at very high energies}

Metagalactic sources of very high energy gamma-rays have been searched for in the SHALON experiment from the very beginning of its operation [12,13]. In 1996, the observations with the SHALON mirror Cherenkov telescope revealed a new metagalactic source of $\gamma$-ray emission at very high energies $E>800 \mathrm{GeV}$ [14, 15] (Figs. 1, 2, 3). The position of emission source detected in our experiment coincides in its coordinates with the Seyfert galaxy NGC $1275[14,15,16,17,18,19$, 20, 21]. NGC 1275 was observed by the SHANON telescope for $271.2 \mathrm{~h}$ in different years (from 1996 to 2012) during the clear moonless nights at zenith angles from $3^{\circ}$ to $33^{\circ}$. The observations were performed using the standard for SHALON technique of obtaining information about the cosmic-ray background and $\gamma$-ray-initiated showers in the same observing session [12, 13, 22, 23]. Gamma-ray emission from NGC 1275 was detected by the SHALON telescope at energies above $800 \mathrm{GeV}$ at the $31.4 \sigma$ confidence level determined according to Li\&Ma [24]. The average integral flux at energies above $800 \mathrm{GeV}$ for NGC 1275 is $I_{N G C 1275}=(7.8 \pm 0.5) \times 10^{-13} \mathrm{~cm}^{-2} \mathrm{~s}^{-1}$ (Fig. 1).

Figure 1 presents the source's image at TeV energies by SHALON experiment. The color scale in Fig. 1 right is in units of the excess above the minimum detected signal for the energy image (in $\mathrm{TeV}$ ). Possible correlations between the emission regions of $\mathrm{TeV} \gamma$-rays and low-energy (radio and X-ray) photons should be established to elucidate the mechanisms of the generation of very high energy emission in the source and to test the models describing them. Figure 3 left
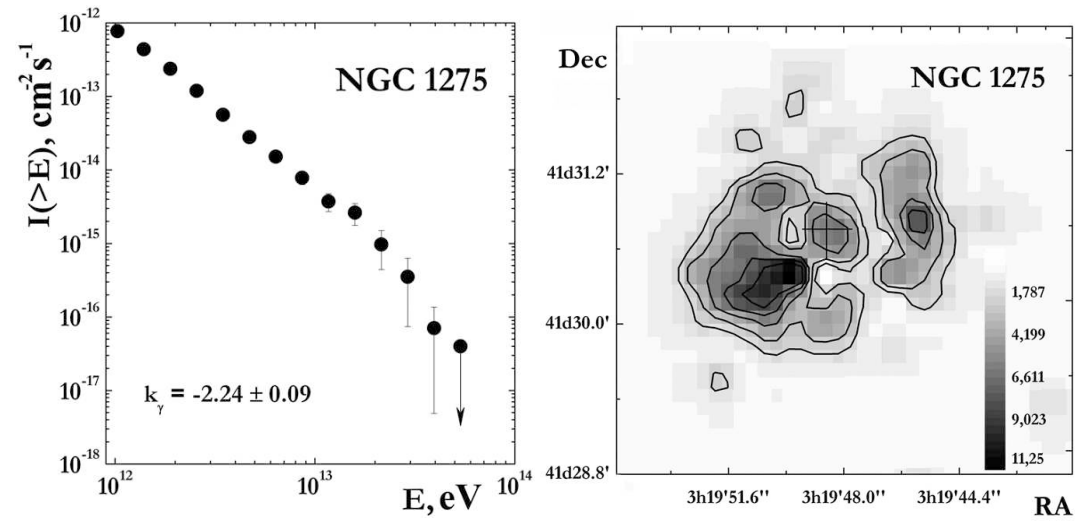

Figure 1: left: Gamma-ray spectrum of NGC 1275 with a power-law index $k_{\gamma}=-2.24 \pm 0.09$. right: The image of the gamma-ray source NGC 1275 at energies $>0.8 \mathrm{TeV}$ by SHALON. 

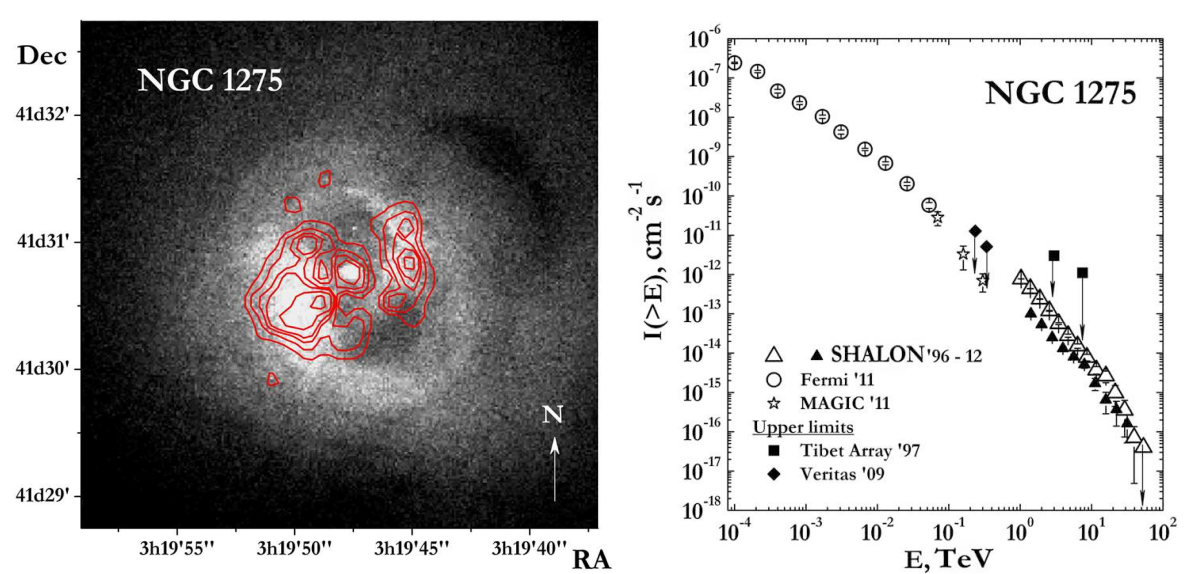

Figure 2: left: Chandra X-ray $(1.5-3.5 \mathrm{keV})$ image of NGC 1275 [25]; the contours indicate the SHALON image of NGC 1275 in the energy range $800 \mathrm{GeV}-40 \mathrm{TeV}$. right: Integral spectrum of high and very high energy $\gamma$-rays from NGC 1275 obtained by SHALON in comparison with the data from the Fermi LAT satellite telescope, the MAGIC and VERITAS Cherenkov telescopes, and the scintillation Tibet Array.

shows a ROSAT X-ray image of NGC 1275 (black-and-white scale); the contours represent the source's radio structure from VLA radio observations. The radio and X-ray emission maxima coincide with the AGN NGC 1275 (Figs. 2, 3). At the same time, the X-ray emission disappears almost completely near the bright areas of the radio components located in the north and the south symmetrically relative to the core [8]. We also combined the SHALON-1 $(0.8-40 \mathrm{TeV})$ and Chandra (1.5 - $3.5 \mathrm{keV}$ X-ray) images. Figure 2 (black-and-white scale) presents a Chandra X-ray $(1.5-3.5 \mathrm{keV})$ image for the central part of the Perseus cluster centered on NGC 1275 with a size of $\sim 5.5 \operatorname{arcmin}[25]$. In the X-ray energy range, the core of the Perseus cluster, on the whole, appears as a clear circularly symmetric structure with a distinct maximum on NGC 1275 (Fig. 2).

The clearly seen dimming in X-ray flux, along with the dip NW of the center, known from the 1979 Einstein observations [26], correlates with the components of the extended double radio structure 3C 84 (Fig. 2). These dips are surrounded by bright (at energies $1.5-3.5 \mathrm{keV}$ ) arc regions from the north and the south. The simplest interpretation is that the intense emission from these rims comes from the shells surrounding the radio lobes [25]. A bright emission spot is also observed to the east.

The emission regions of very high energy $\gamma$-rays observed by SHALON from NGC 1275 have a structure similar to that described above (see [25]) and well correlates with the photon emission regions in the energy range $1.5-3.5 \mathrm{keV}$ (Fig. 2). A correlation of the emission with energies $0.8-40 \mathrm{TeV}[17,18,19]$ and the X-ray emission in the range $0.3-7 \mathrm{keV} \mathrm{[25]} \mathrm{was} \mathrm{also} \mathrm{found.}$ Thus, the TeV $\gamma$-ray emission recorded by SHALON from NGC 1275 has an extended structure with a distinct core centered at the source's position.

To analyze the emission related to this core, we additionally identified the emission component corresponding to the central region of NGC 1275 with a size of 32". The emission from the central region of NGC 1275 was detected at energies above $0.8 \mathrm{TeV}$ at a $13.5 \sigma$ confidence level determined by the Li\&Ma method [24] with a average integral flux $I(>800 \mathrm{GeV})=(3.26 \pm$ $0.30) \times 10^{-13} \mathrm{~cm}^{-2} \mathrm{~s}^{-1}$. The $\gamma$-ray energy spectrum of the central component in the entire energy range from 0.8 to $40 \mathrm{TeV}$ is well described by a power law with an exponential cutoff, 

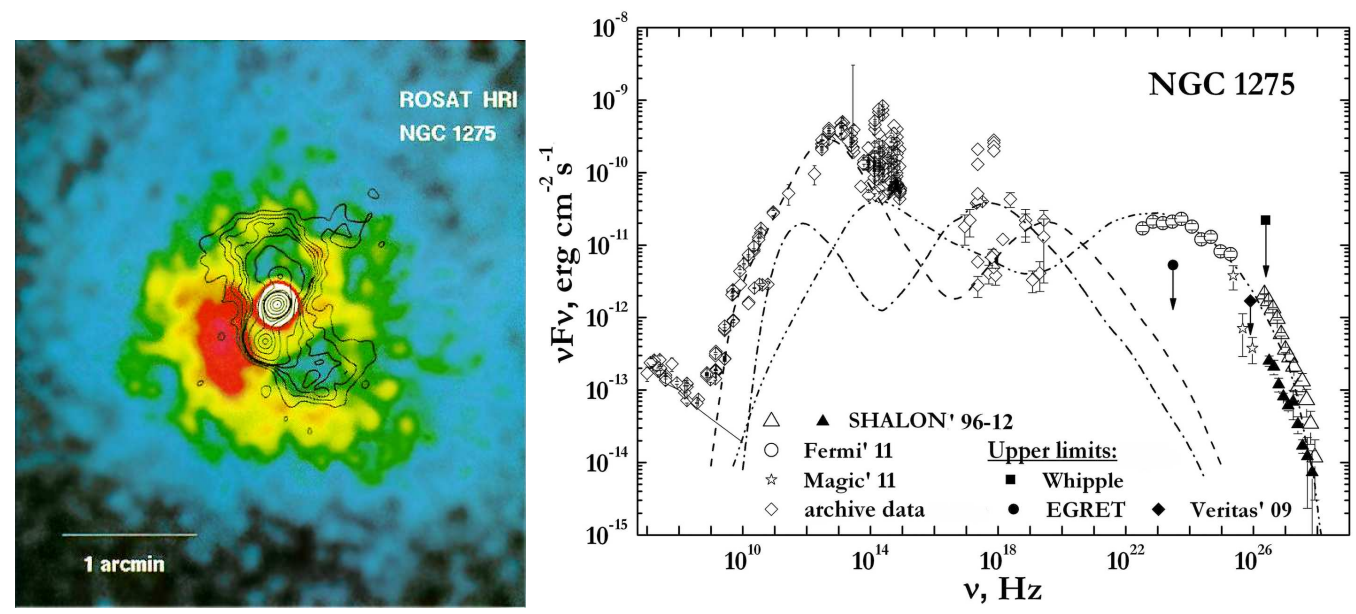

Figure 3: left: ROSAT X-ray $(0.1-2.4 \mathrm{keV})$ image of NGC 1275 [8]. The contours represent the source's radio structure from VLA radio observations. right: Spectral energy distribution of the $\gamma$-ray emission from NGC 1275. $\triangle$ and black triangle represent the data from the SHALON Cherenkov telescope. $\bigcirc$ represent the Fermi LAT [27]; stars are Magic [28] data. The arrows indicate the upper limits from EGRET [29], Whipple [30], and VERITAS[31] data (see the text). The dashed, dash-dotted, and dash-dotted with two dots curves indicate the spectral energy distributions of NGC 1275 obtained in the CM model [32].

$I\left(>E_{\gamma}\right)=(2.92 \pm 0.11) \times 10^{-13} \times E^{-1.55 \pm 0.10} \times \exp \left(-E_{\gamma} / 10 T e V\right) \mathrm{cm}^{-2} s^{-1}$ (see Fig. 2, the black triangles). If the source's spectrum in the energy range $0.8-20 \mathrm{TeV}$ is described by a simple power law $F\left(E_{0}>0.8 T e V\right) \propto E^{k_{\gamma}}$, the spectral index is $k_{\gamma}=-1.92 \pm 0.11$. The SHALON spectrum corresponding to the emission from the central region of NGC 1275 is represented in Fig. 2 by the black triangles.

Recently, the AGN NGC 1275 was also recorded by the MAGIC ground-based mirror Cherenkov telescope at energies above $100 \mathrm{GeV}$ in the 2010 - 2011 observations [33]. Figure 2 compares the integral $\gamma$-ray spectrum of NGC 1275 and its central region obtained from SHALON data (1996 2012) with the Fermi LAT (2009 - 2011) [34] and MAGIC (2010 - 2011) [33] experimental data.

\section{Variability of the gamma-ray emission from NGC 1275}

Reliably revealing flares and their duration in long-term observations with mirror Cherenkov telescopes is complicated by the fact that the technique makes a continuous tracking of the source impossible, because it requires such conditions as moonless nights, which already creates a gap in the data for more than 10 days; an ideal atmosphere without clouds and haze and, in addition, the source's passage at a distance of no more than $35^{\circ}$ from zenith are needed, because the influence of a change in atmospheric thickness should be minimal.

Nevertheless, revealing correlations between the emissions in different energy ranges, comparing the emission regions, and, in particular, the detection of the flux changes remains necessary, because it makes it possible to judge the nature of the source, its evolution, and the emission generation mechanisms in various objects.

The observed $\gamma$-ray flux variations, on average, do not exceed $20 \%$ of $(7.8 \pm 0.5) \times 10^{-13} \mathrm{~cm}^{-2} \mathrm{~s}^{-1}$. The SHALON mirror Cherenkov telescope has detected three short-time (within five days) increases and one decrease of the very high energy $\gamma$-ray flux in the entire time of observations of NGC 1275. Given these variations, the flux decrease below the average was recorded in 1999 and 

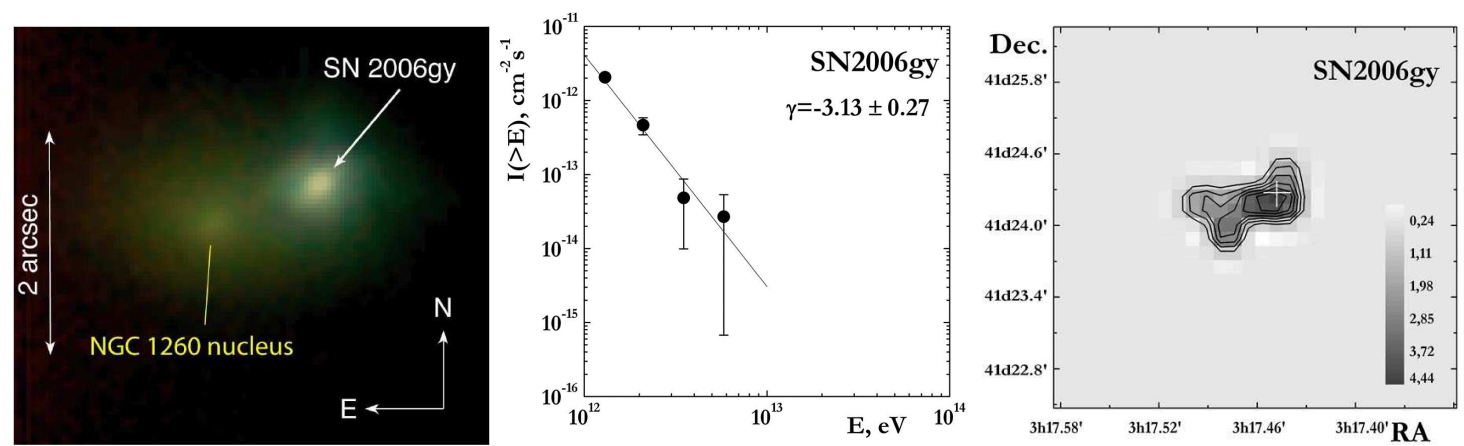

Figure 4: left: The image of SN2006 gy and the nucleus of NGC 1260 at three wavebands: J band(1.25 $\mu \mathrm{m}), \mathrm{H}$ band $(1.65 \mu \mathrm{m})$, and Ks band $(2.2 \mu \mathrm{m})$ [35]. The SN2006gy by SHALON: center: the $\gamma$-quantum integral spectrum; right: The SN2006gy image at TeV energy range.

the integral flux was $(4.7 \pm 1.3) \times 10^{-13} \mathrm{~cm}^{-2} \mathrm{~s}^{-1}$. The increases were detected in late Jan. 2001, late Nov. early Dec. 2005, and late Oct. 2009. The fluxes in these periods were $(21.2 \pm 7.5) \times 10^{-13}$ , $(35.5 \pm 12.4) \times 10^{-13}$, and $(23.4 \pm 4.5) \times 10^{-13} \mathrm{~cm}^{-2} \mathrm{~s}^{-1}$, respectively. The duration of the flux increase in October 2009 was 3 days. No intervals of flux increase were found in 2001 and 2005, because the observations were interrupted due to weather conditions in both cases.

To reveal possible correlations of the emissions in various energy ranges, including those at high and very high energies, we compared the NGC $1275 \gamma$-ray fluxes by SHALON in the periods when the observations were simultaneous with the ones by the Fermi LAT experiment. The published Fermi LAT data were obtained from August 4, 2008, to September 30, 2010 [34]. The SHALON observations of NGC 1275 were performed in November 2008 with a break for the Moon's time, October 2009, and mid-November-early December 2010. In this time, only one $\gamma$-ray flux increase to $(23.4 \pm 4.5) \times 10^{-13} \mathrm{~cm}^{-2} \mathrm{~s}^{-1}$ was detected in the period of October 18 - 20, 2009 . These periods of SHALON observations do not coincide with the times of the main flares observed at Fermi LAT [34]. A slight local flux increase can be seen in the period of mid-October 2009 [34], which corresponds to the above-mentioned $\gamma$-ray flux increase observed by SHALON.

\section{SN2006 gy}

The flux increase was detected from the region NGC 1275 in autumn 2006. The detailed analysis of $\gamma$-shower direction turned out the detection of metagalactic object. This object was identified with the supernova SN2006 gy [36] (fig. 4)that is about 10 minutes away from NGC 1275.

Observations had been done in cloudless nights of moonless periods of September, October, November, December 2006 and then during the winter of 2007. No flux increase was found in September observations. In the flare, observed on October 22, the flux increased about 6 times from the NGC 1275 average flux and stayed on this level all October moonless period. After the standard analysis, a excess corresponding to a $6.2 \sigma$ [24] was determined. The integral $\gamma$-ray flux for SN 2006gy is found to be $(3.71 \pm 0.65) \times 10^{-12} \mathrm{~cm}^{-2} \mathrm{~s}^{-1}$ [37] at energies of $>0.8 \mathrm{TeV}$. The energy spectrum of SN2006 gy at 0.8 to $7 \mathrm{TeV}$ can be approximated by the power law $F\left(>E_{O}\right) \propto E^{k_{\gamma}}$, with $k_{\gamma}=-3.13 \pm 0.27$ (fig. 4). An image of $\gamma$-ray emission from SN2006 gy by SHALON telescope is shown in Fig. 4. Follow-up observations on end of November showed that the flux of SN2006 gy had dropped to a flux level of about $(0.69 \pm 0.17) \times 10^{-12} \mathrm{~cm}^{-2} \mathrm{~s}^{-1}$ and was constant during the November, December period. The results of observation analysis of 2007 have no revealed 

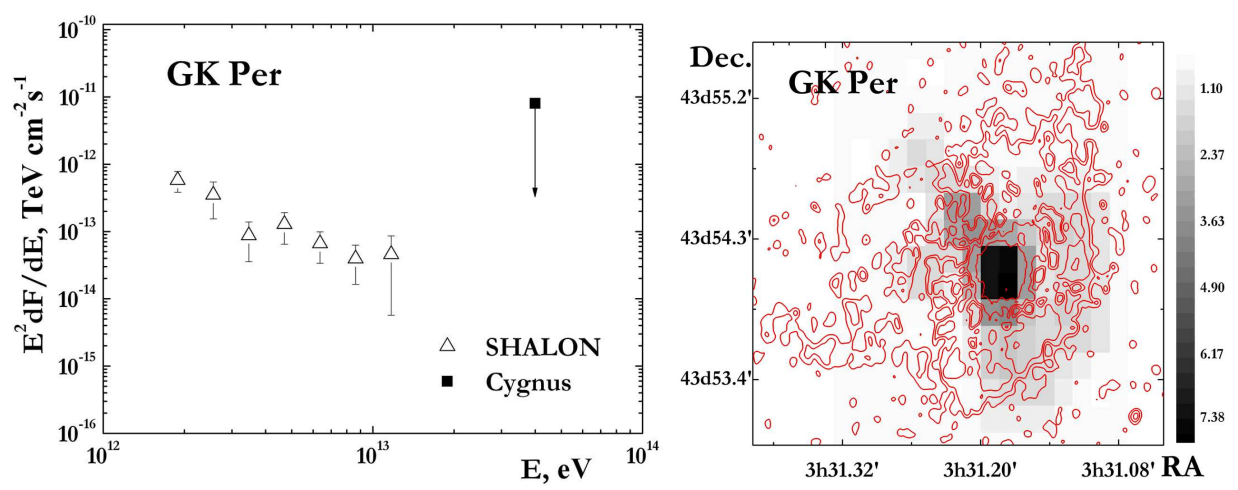

Figure 5: left: Spectral energy distribution from GK Per obtained by SHALON. right: The image of GK Per in the energy range $800 \mathrm{GeV}-40 \mathrm{TeV}$ by SHALON the contours indicate Chandra X-ray $(1.5-3.5$ $\mathrm{keV}$ ) image of GK Per [38].

$\mathrm{TeV} \gamma$-ray emission from region of SN 2006gy. So, the explosion of extragalactic supernova was observed at $\mathrm{TeV}$ energies for the first time with SHALON Cherenkov telescope.

\section{GK Per (Nova 1901)}

During the observations of NGC 1275 the SHALON field of view contains the source of nonthermal radio and X-ray emission GK Per (Nova 1901) of classical nova type as it located at $\sim 3^{\circ}$ SW from NGC 1275. So due to the large telescopic field of view $\left(\geq 8^{\circ}\right)$ the observations of NGC 1275 is naturally followed by the tracing of GK Per. GK Per as a source accompanying to NGC 1275 was observed with SHALON telescope during the period from 1996y to 2012y till now for a total of 111 hours. The $\gamma$-ray source associated with the GK Per was detected above $800 \mathrm{GeV}$ with average $\gamma$-flux $I_{G K P e r}(>0.8 T e V)=(2,9 \pm 1,3) \times 10^{-13} \mathrm{~cm}^{-2} s^{-1}$. The $\gamma$-ray source associated with the GK Per was detected above $800 \mathrm{GeV}$ with a statistical significance[24] of 9.2 $\sigma$. The signal significance for this SNR is less then one for the source with similar flux and spectrum index obtained in the same observation hours because of less collection field of view relative to the standard procedure of SHALON experiment. The corrections for the effective field of view were made to calculate source flux and energy spectrum. The energy spectrum of $\gamma$-rays in the observed energy region from 2 to $15 \mathrm{TeV}$ (Fig. 5, left) is well described by the power law $F\left(>E_{O}\right) \propto E^{k_{\gamma}}$, with $k_{\gamma}=-1.90 \pm 0.35$. The image of GK Per at TeV-energies by SHALON are shown with Fig. 5, right. The analysis of $\gamma$-ray shower arrival direction revealed the main $\mathrm{TeV}$-emission region coinciding with the position of central source of GK Per and the weak emission of shell, that is also observed in X-ray by Chandra [38].

\section{NGC 1275 as a point and extended source}

As has been pointed out above, the Perseus cluster of galaxies with the central galaxy NGC 1275 is ideally suitable both for studying the physics of relativistic jets from AGNs and for revealing the feedback role of the central galaxy. Evidence for the latter was obtained in ROSAT and Chandra observations at low energies, from which shells of hot gas and cavities that spatially coincide with the radio structures originating in the central, active part of the AGN can be seen (Fig. 3, left and [8, 9, 25, 39]. The observational data for NGC 1275 at energies $800 \mathrm{GeV}-40 \mathrm{TeV}$, namely the images of the galaxy and its surroundings (Figs. 1 and 2), as well as the flux variations suggest that the $\mathrm{TeV} \gamma$-ray emission in these regions is produced by a number of processes. 
The extended structure around NGC 1275 (Fig. 2) that spatially coincides with the X-ray emission regions (Fig. 2) can be produced by mechanisms related to the generation of an X-ray structure $[9,25,39]$. The brightness distribution of the X-ray emission and the observed $\mathrm{TeV}$ emission shows a sharp increase in intensity right outside the bubbles blown by the central black hole and visible in the radio band. This suggests that the X-ray-generating particles are swept up from the region of the radio lobes under the pressure of cosmic rays and magnetic fields generated in the jets at the center of NGC $1275[9,39]$. The structures visible in TeV $\gamma$-rays are formed through the interaction of very high energy cosmic rays with the gas inside the Perseus cluster and interstellar gas heating at the boundary of the bubbles blown by the central black hole in NGC 1275 .

The presence of emission in the energy range $1-40 \mathrm{TeV}$ from a central region of $\sim 32$ " in size around the nucleus of NGC 1275 (see Fig. 2, the black triangles) and the short-time flux variability point to the origin of the very high energy emission as a result of the generation of jets ejected by the central supermassive black hole of NGC 1275. The multifrequency spectral energy distribution for the nucleus of NGC 1275, up to high and very high energies, was described in the CM model [32] and is a composition of the components of inverse Compton scattering of the intrinsic synchrotron radiation from relativistic electrons (synchrotron self-Compton) of three separate plasma blobs ejected from the inner regions of the NGC 1275 nucleus (Fig. 3, the dashed, dash-dotted, and dash-dotted with two dots curves). The available Fermi LAT data at high energies and the SHALON observations at very high energies in a region < 32" around NGC 1275 are described in terms of this model with one of the components produce synchrotron self-Compton emission of the relativistic jets from the nucleus itself (Fig. 3, the dash-dotted with two dots curve).

\section{Conclusion}

Long-term studies of the central galaxy in the cluster, NGC 1275, are being carried out in the SHALON experiment. We presented the results of fifteen-year-long observations of the AGN NGC 1275 at energies $800 \mathrm{GeV}-40 \mathrm{TeV}$ discovered by the SHALON telescope in $1996[14,15$, $16,17,18,19,20]$. The data obtained at very high energies, namely the images of the galaxy and its surroundings, and the flux variability indicate that the $\mathrm{TeV}$ gamma-ray emission is generated by a number of processes: in particular, part of this emission is generated by relativistic jets in the nucleus of NGC 1275 itself. Whereas, the presence of an extended structure around NGC 1275 is evidence of the interaction of cosmic rays and magnetic fields generated in the jets at the galactic center with the gas of the Perseus cluster. Also, in the long-term observations of the Perseus Cluster revealed the $\gamma$-ray emission from the two of nearby object - GK Per of classical nova type, located at $\sim 3^{\circ} \mathrm{SW}$ from NGC 1275 and the explosion of extragalactic supernova SN2006gy that is about 10 minutes away from NGC 1275. So, the explosion of extragalactic supernova was observed at $\mathrm{TeV}$ energies for the first time with SHALON Cherenkov telescope.

\section{References}

[1] B. Dennison, Astrophys. J. 239, L93 (1980).

[2] B. P. Houston, A. W. Wolfendale, and E. C. M. Young, J. Phys. G: Nucl. Phys. 10, L147 (1984).

[3] S. Colafrancesco and P. Blasi, Astropart. Phys. 9, 227 (1998).

[4] C. L. Sarazin, Astrophys. J. 520, 529 (1999). 
[5] F. Miniati, T. W. Jones, H. Kang, et al., Astrophys. J.62, 233 (2001).

[6] A. N. Timokhin, F. A. Aharonian, and A. Yu. Neronov, Astron. Astrophys. 417, 391 (2004).

[7] S. Colafrancesco and P. Marchegiani, Astron. Astrophys. 502, 711 (2009).

[8] H. Böhringer, W. Voges, A. C. Fabian, et al., Mon. Not. R. Astron. Soc. 264, L25 (1993).

[9] A. C. Fabian, J. S. Sanders, G. B. Taylor, et al., Mon. Not. R. Astron. Soc. 366, 417 (2006).

[10] J. S. Gallagher, Astron. Nachr. 220, 1040G (2009).

[11] M. Strauss, J. P. Huchra, M. Davis, et al., Astrophys. J. Suppl. Ser. 83, 29S (1992).

[12] V. G. Sinitsyna, in Proceedings of the Workshop, Towards a Major Atmospheric Cherenkov Detector IV, Ed. by M. Cresti (Univ. of Padova, Padova, 1995), p. 133.

[13] V. G. Sinitsyna, Nuovo Cim. 19C, 965 (1996).

[14] V. G. Sinitsyna, in Proceedings of the Workshop, Towards a Major Atmospheric Cherenkov Detector V, Kruger Park, South Africa, Ed. by O. De Jager (Westprint, Potchefstroom, 1997), p. 136.

[15] V. G. Sinitsyna, AIP Conf. Proc. 515, 293 (2000).

[16] V. G. Sinitsyna, Rad. Phys. Chem. 75, 880 (2006).

[17] V. G. Sinitsyna, S. I. Nikolsky, V. Y. Sinitsyna, et al., Nucl. Phys. B (Proc. Suppl.) 122, 409 (2003).

[18] V. G. Sinitsyna, S. I. Nikolsky, V. G. Sinitsyna, et al., Bull. Russ. Acad. Sci. 71, 906 (2007).

[19] V. G. Sinitsyna, F. I. Musin, S. I. Nikolsky, et al., Nucl. Phys. B (Proc. Suppl.) 196, 442 (2009).

[20] S. I. Nikolsky and V. G. Sinitsyna, Phys. At. Nucl. 67, 1900 (2004).

[21] V. G. Sinitsyna, S. I. Nikolsky, V. Y. Sinitsyna, et al., Nucl. Phys. B (Proc. Suppl.) 75A, 352 (1999).

[22] V. G. Sinitsyna and V. Yu. Sinitsyna, Kratk. Soobshch. Fiz. 4, 1 (2011).

[23] V. G. Sinitsyna and V. Yu. Sinitsyna, Astron. Lett. 37(9), 621 (2011).

[24] T.-P. Li and Y.-Q. Ma, Astrophys. J. 272, 317 (1983).

[25] A. C. Fabian, J. S. Sanders, S. W. Allen, et al., Mon. Not. R. Astron. Soc. 318, L65 (2000).

[26] A. C. Fabian, E. M. Hu, L. L. Cowie, et al., Astrophys. J. 248, 47 (1981).

[27] A. A. Abdo, M. Ackermann, M. Ajello, et al., Astrophys. J. 699, 31 (2009).

[28] J. Aléksic, L. A. Antonelli, P. Antoranz, et al., Astrophys. J. 710, 634A (2010).

[29] D. J. Thompson, D. L. Bertsch, B. L. Dingus, et al., Astrophys. J. Suppl. 101, 209 (1995).

[30] J. S. Perkins, H. M. Badran, G. Blaylock, et al., Astrophys. J. 644, 148 (2006).

[31] V. A. Acciari, E. Aliu, T. Arlen, et al., Astrophys. J. 706, L275 (2009).

[32] S. Colafrancesco, P. Marchegiani and P. Giommi, Astron. Astrophys. 519, A82 (2010).

[33] J. Aléksic, E. A. Alvarez, L. A. Antonelli, et al., Astron. Astrophys. 539, L2 (2012).

[34] A. M. Brown and J. Adams, Mon. Not. R. Astron. Soc. 413, 2785 (2011).

[35] N. Smith et al. Astrophys.J., 666, 1116 (2007).

[36] E. O. Ofek, P. B. Cameron, et. al., arXiv:astro-ph/0612408v1

[37] V.G. Sinitsyna et al., J. Phys. Soc. Jpn., Suppl. A 78, 192 (2009).

[38] S. Balman Astrophys. J., 627, 933 (2005).

[39] E. Churazov, W. Forman, C. Jones, H. Böhringer, Astron. Astrophys. 356, 788 (2000). 